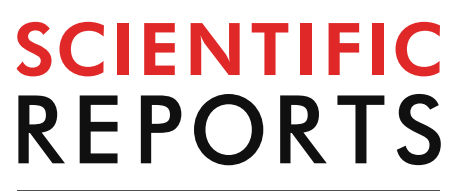

natureresearch

Check for updates

\title{
Machine learning improves the prediction of febrile neutropenia in Korean inpatients undergoing chemotherapy for breast cancer
}

\author{
Bum-Joo Cho ${ }^{1}$, Kyoung Min Kim², Sanchir-Erdene Bilegsaikhan ${ }^{3}$ \& Yong Joon Suh ${ }^{4}$
}

Febrile neutropenia (FN) is one of the most concerning complications of chemotherapy, and its prediction remains difficult. This study aimed to reveal the risk factors for and build the prediction models of FN using machine learning algorithms. Medical records of hospitalized patients who underwent chemotherapy after surgery for breast cancer between May 2002 and September 2018 were selectively reviewed for development of models. Demographic, clinical, pathological, and therapeutic data were analyzed to identify risk factors for $\mathrm{FN}$. Using machine learning algorithms, prediction models were developed and evaluated for performance. 0 f 933 selected inpatients with a mean age of $51.8 \pm 10.7$ years, $\mathrm{FN}$ developed in $409(43.8 \%)$ patients. There was a significant difference in $\mathrm{FN}$ incidence according to age, staging, taxane-based regimen, and blood count 5 days after chemotherapy. The area under the curve (AUC) built based on these findings was 0.870 on the basis of logistic regression. The AUC improved by machine learning was 0.908 . Machine learning improves the prediction of $\mathrm{FN}$ in patients undergoing chemotherapy for breast cancer compared to the conventional statistical model. In these high-risk patients, primary prophylaxis with granulocyte colony-stimulating factor could be considered.

Chemotherapy-induced febrile neutropenia $(\mathrm{FN})$ is one of the most concerning complications in patients with breast cancer undergoing chemotherapy ${ }^{1}$. Neutropenia is a principal dose-limiting toxicity of myelosuppressive chemotherapy that predisposes patients to grave infections ${ }^{2}$. Moreover, infection in patients with neutropenia is the direct consequence of chemotherapy-induced neutropenia ${ }^{3}$. Chemotherapy-induced neutropenia is a principal risk factor for infection-related morbidity ${ }^{4}$. Further, mortality rates related with FN vary from 2 to $21 \%^{5,6}$.

Chemotherapy-induced FN commonly occurs during the initial cycle of cytotoxic therapy and increases in frequency with both duration and depth of the neutropenia ${ }^{3}$. In addition to an influence on quality of life, chemotherapy-induced FN exposes patients with cancer to life-threatening infections. Considering the severity of FN, the most patients who develop FN are hospitalized for evaluation and injected with broad-spectrum antibiotics. Along with infections, chemotherapy-induced FN frequently results in dose reductions and treatment delays which have been known to compromise treatment ${ }^{1,7}$. The risk of developing FN appears to depend on diverse factors, including patient-related factors, tumor burden, and chemotherapy regimen ${ }^{6}$.

Thus, primary prevention, through the administration of granulocyte colony-stimulating factor (G-CSF), is recommended by guidelines when a significant risk of FN exists ${ }^{4,8,9}$. G-CSF stimulates the maturation, proliferation, and release of neutrophils, leading to a dose-dependent increase in circulating neutrophils ${ }^{10}$. Primary prophylaxis with G-CSF decreased the risk of FN by $50 \%$ in patients with solid tumors without altering tumor response, overall survival, or infection-related mortality ${ }^{8}$. Currently, the criteria for the use of G-CSF and other means to reduce the risk of FN are based on low-quality evidence ${ }^{11}$.

${ }^{1}$ Department of Ophthalmology, Hallym University Sacred Heart Hospital, Anyang, Korea. ${ }^{2}$ Institute of New Frontier Research, Hallym University College of Medicine, Chuncheon, Korea. ${ }^{3}$ Department of Biomedical Science, Hallym University, Chuncheon, Korea. ${ }^{4}$ Department of Breast and Endocrine Surgery, Hallym University Sacred Heart Hospital, 22, Gwanpyeong-ro 170 beon-gil, Dongan-gu, Anyang 14068, Korea. ${ }^{凶}$ email: nicizm@gmail.com 


\begin{tabular}{|c|c|c|c|}
\hline Parameters & FN group $(n=366)$ & non-FN group $(n=477)$ & $p$ value \\
\hline Age (years), means $\pm S D$ & & & 0.004 \\
\hline$\leq 50$ & $157(42.9)$ & $276(57.9)$ & $<0.001$ \\
\hline$>50$ & $209(57.1)$ & $201(42.1)$ & \\
\hline Body surface area $\left(\mathrm{m}^{2}\right)$, means $\pm \mathrm{SD}$ & $1.58 \pm 0.14$ & $1.57 \pm 0.13$ & 0.498 \\
\hline Hypertension, n (\%) & $112(30.6)$ & $113(23.7)$ & 0.028 \\
\hline Diabetes mellitus, n (\%) & $42(11.5)$ & $44(9.2)$ & 0.303 \\
\hline Tuberculosis, n (\%) & $9(2.5)$ & $14(2.9)$ & 0.832 \\
\hline Breast-conserving surgery, n (\%) & $201(55.1)$ & $345(72.3)$ & $<0.001$ \\
\hline Tumor size $(\mathrm{cm})$, mean $\pm \mathrm{SD}$ & $2.7 \pm 2.0$ & $2.3 \pm 1.4$ & 0.002 \\
\hline Positive lymph node, means \pm SD & $2.6 \pm 5.2$ & $1.2 \pm 3.4$ & $<0.001$ \\
\hline $\mathrm{ER}, \mathrm{n}(\%)$ & $268(74.0)$ & $312(66.0)$ & 0.012 \\
\hline $\mathrm{PR}, \mathrm{n}(\%)$ & $225(62.2)$ & $280(59.2)$ & 0.392 \\
\hline Her-2, n (\%) & $108(29.5)$ & $133(27.9)$ & 0.645 \\
\hline CA $15-3$, means \pm SD & $56.3 \pm 202.0$ & $24.0 \pm 136.5$ & 0.009 \\
\hline TNM staging, $\mathrm{n}(\%)$ & & & $<0.001$ \\
\hline $\mathrm{I} / \mathrm{II}$ & $248(67.8)$ & $410(86.0)$ & \\
\hline III/IV & $118(32.2)$ & $67(14.0)$ & \\
\hline Taxane-based regimen, $\mathrm{n}(\%)$ & $245(66.9)$ & $184(38.6)$ & $<0.001$ \\
\hline \multicolumn{4}{|c|}{ CBC (before chemotherapy), means \pm SD } \\
\hline Hemoglobin $(\mathrm{g} / \mathrm{dL})$ & $12.9 \pm 1.2$ & $13.0 \pm 1.4$ & 0.426 \\
\hline Platelet $\left(\times 10^{3} / \mu \mathrm{L}\right)$ & $262 \pm 63$ & $269 \pm 65$ & 0.168 \\
\hline Neutrophil $\left(\times 10^{3} / \mu \mathrm{L}\right)$ & $3.725 \pm 1.550$ & $3.815 \pm 1.420$ & 0.383 \\
\hline Lymphocyte $\left(\times 10^{3} / \mu \mathrm{L}\right)$ & $1.905 \pm 0.603$ & $1.971 \pm 0.621$ & 0.119 \\
\hline \multicolumn{4}{|c|}{ CBC (5 days after chemotherapy), means \pm SD } \\
\hline Hemoglobin $(g / d L)$ & $10.9 \pm 1.7$ & $11.5 \pm 1.1$ & $<0.001$ \\
\hline Platelet $\left(\times 10^{3} / \mu \mathrm{L}\right)$ & $221 \pm 82$ & $226 \pm 62$ & 0.378 \\
\hline Neutrophil $\left(\times 10^{3} / \mu \mathrm{L}\right)$ & $3.329 \pm 2.278$ & $3.067 \pm 1.343$ & 0.052 \\
\hline Lymphocyte $\left(\times 10^{3} / \mu \mathrm{L}\right)$ & $0.867 \pm 0.374$ & $1.561 \pm 0.593$ & $<0.001$ \\
\hline SERM, n (\%) & $139(38.0)$ & $221(46.3)$ & 0.017 \\
\hline LHRH, n (\%) & $112(30.6)$ & $190(39.8)$ & 0.006 \\
\hline Aromatase inhibitor, n (\%) & $148(40.4)$ & $121(25.4)$ & $<0.001$ \\
\hline Radiation treatment, n (\%) & $292(79.8)$ & $401(84.1)$ & 0.122 \\
\hline Herceptin, n (\%) & $101(27.6)$ & $130(27.3)$ & 0.938 \\
\hline
\end{tabular}

Table 1. Clinical demographic characteristics of patients with and without febrile neutropenia in the training dataset. $F N$ febrile neutropenia, $S D$ standard deviation, $B S A$ body surface area, $E R$ estrogen receptor, $P R$ progesterone receptor, Her-2 human epidermal growth factor receptor 2, $C A$ cancer antigen, $C B C$ complete blood count, SERM selective estrogen receptor modulator, $L H R H$ luteinizing hormone-releasing hormone, $F / U$ follow-up.

Trials to prevent FN events during chemotherapy administration require an evaluation of risk factors related with the development of critical neutropenia ${ }^{12}$. However, this evaluation remains inaccurate ${ }^{3}$. Until now, no available prediction model has gained general acceptance ${ }^{12}$. A particularly important field of uncertainty that is emphasized by these findings is the absence of risk prediction models that estimate the risk of FN in patients reliably ${ }^{8}$.

Machine learning techniques have been widely adopted for the investigation of biomedical big data over the past years ${ }^{13}$. Recently, machine learning frameworks known as deep learning, which are based on artificial neural networks, have attracted more attention because of its notable success in predicting clinical outcomes of interest ${ }^{14}$. In this study, we aimed to unravel the predictive factors for and improve the prediction of FN by machine learning.

\section{Results}

Of the 933 patients, the mean age was $51.8 \pm 10.7$ years. 611 (65.5\%) patients underwent breast-conserving surgery. Regarding staging, 737 (79.0\%) patients were staged as I/II, while $196(21.0 \%)$ patients were staged as III/ IV. The median length of follow-up was $4.9 \pm 2.9$ years. FN developed in 409 (43.8\%) patients, and the period until the development of FN was $10.2 \pm 2.8$ days.

In the training dataset, 843 patients were grouped according to the presence of FN. Patients with and without FN are compared in Table 1. There was a significant difference in the incidence of FN according to age, staging, and taxane-based regimen. The group with FN was older, had advanced disease, and received taxane-based regimens more frequently. Differences between the FN and non-FN groups were also found in complete blood 


\begin{tabular}{|c|c|c|c|}
\hline Parameters & FN group $(n=43)$ & non-FN group $(n=47)$ & $p$ value \\
\hline Age (years), means $\pm S D$ & & & 0.050 \\
\hline$\leq 50$ & $13(30.2)$ & $24(51.1)$ & \\
\hline$>50$ & $30(69.8)$ & $23(48.9)$ & \\
\hline Body surface area $\left(\mathrm{m}^{2}\right)$, means $\pm \mathrm{SD}$ & $1.61 \pm 0.14$ & $1.63 \pm 0.14$ & 0.383 \\
\hline Hypertension, n (\%) & $12(27.9)$ & $11(23.4)$ & 0.638 \\
\hline Diabetes mellitus, n (\%) & $5(11.6)$ & $5(10.6)$ & 1.000 \\
\hline Tuberculosis, n (\%) & $0(0)$ & $0(0)$ & 1.000 \\
\hline Breast-conserving surgery, n (\%) & $28(65.1)$ & $37(78.7)$ & 0.166 \\
\hline Tumor size $(\mathrm{cm})$, mean $\pm \mathrm{SD}$ & $2.7 \pm 1.3$ & $2.5 \pm 1.2$ & 0.490 \\
\hline Positive lymph node, means \pm SD & $1.7 \pm 4.0$ & $0.3 \pm 1.4$ & 0.038 \\
\hline $\mathrm{ER}, \mathrm{n}(\%)$ & $34(79.1)$ & $26(55.3)$ & 0.046 \\
\hline $\mathrm{PR}, \mathrm{n}(\%)$ & $26(60.5)$ & $18(38.3)$ & 0.057 \\
\hline Her-2, n (\%) & $12(27.9)$ & $7(14.9)$ & 0.196 \\
\hline CA $15-3$, means \pm SD & $15.2 \pm 12.0$ & $11.7 \pm 7.8$ & 0.140 \\
\hline TNM staging, n (\%) & & & 0.003 \\
\hline I/II & $33(76.7)$ & $46(97.9)$ & \\
\hline III/IV & $10(23.3)$ & $1(2.1)$ & \\
\hline Taxane-based regimen, $\mathrm{n}(\%)$ & $23(53.5)$ & $14(29.8)$ & 0.032 \\
\hline \multicolumn{4}{|c|}{ CBC (before chemotherapy), means \pm SD } \\
\hline Hemoglobin $(\mathrm{g} / \mathrm{dL})$ & $13.0 \pm 1.6$ & $13.1 \pm 1.2$ & 0.940 \\
\hline Platelet $\left(\times 10^{3} / \mu \mathrm{L}\right)$ & $263 \pm 56$ & $265 \pm 77$ & 0.590 \\
\hline Neutrophil $\left(\times 10^{3} / \mu \mathrm{L}\right)$ & $3.661 \pm 1.574$ & $4.006 \pm 1.683$ & 0.550 \\
\hline Lymphocyte $\left(\times 10^{3} / \mu \mathrm{L}\right)$ & $2.024 \pm 0.575$ & $2.193 \pm 0.640$ & 0.250 \\
\hline \multicolumn{4}{|c|}{ CBC (5 days after chemotherapy), means \pm SD } \\
\hline Hemoglobin $(\mathrm{g} / \mathrm{dL})$ & $10.7 \pm 1.1$ & $5.314 \pm 1.593$ & 0.017 \\
\hline Platelet $\left(\times 10^{3} / \mu \mathrm{L}\right)$ & $219 \pm 99$ & $11.3 \pm 1.0$ & 0.170 \\
\hline Neutrophil $\left(\times 10^{3} / \mu \mathrm{L}\right)$ & $3.977 \pm 3.468$ & $3.348 \pm 1.208$ & 0.220 \\
\hline Lymphocyte $\left(\times 10^{3} / \mu \mathrm{L}\right)$ & $0.838 \pm 0.339$ & $1.703 \pm 0.683$ & $<0.001$ \\
\hline SERM, n (\%) & $13(30.2)$ & $23(48.9)$ & 0.087 \\
\hline LHRH, n (\%) & $11(25.6)$ & $20(42.6)$ & 0.121 \\
\hline Aromatase inhibitor, n (\%) & $22(51.2)$ & $14(29.8)$ & 0.053 \\
\hline Radiation treatment, $\mathrm{n}(\%)$ & $35(81.4)$ & $41(87.2)$ & 0.564 \\
\hline Herceptin, $\mathrm{n}(\%)$ & $13(30.2)$ & $10(21.3)$ & 0.346 \\
\hline
\end{tabular}

Table 2. Clinical demographic characteristics of patients with and without febrile neutropenia in the testing dataset. FN febrile neutropenia, $S D$ standard deviation, $E R$ estrogen receptor, $P R$ progesterone receptor, Her- 2 human epidermal growth factor receptor 2, $C A$ cancer antigen, $C B C$ complete blood count, SERM selective estrogen receptor modulator, $A I$ aromatase inhibitor, $L H R H$ luteinizing hormone-releasing hormone, $F / U$ follow-up.

count/differential blood count 5 days after chemotherapy. Lymphocyte count was significantly lower in the group with FN. We calculated and validated this predictive model using the testing dataset. The demographic characteristic of the 90 patients in the testing dataset are presented in Table 2 . The highest AUC value was 0.870 on the basis of logistic regression.

Factors associated with FN were selected by machine learning algorithms. The performances of prediction models in the testing dataset are presented in Table 3. XGboosting showed the best performance with an AUC of 0.908 . The AUC of each algorithm is presented in Fig. 1. Data collected for hierarchical levels were used as input data for the decision tree model. The root node of the decision tree was lymphocyte count 5 days after chemotherapy, and the cut-off was $0.982\left(\times 10^{3} / \mu \mathrm{L}\right)($ Fig. 2$)$.

\section{Discussion}

In the present study, multivariate analysis demonstrated predictive factors for FN, including age, staging, and taxane-based regimen. The lymphocyte count 5 days after chemotherapy was also a strong predictive factor for FN. Based on these findings, logistic regression showed an AUC of 0.870 for validation. Even in machine learning, the lymphocyte count 5 days after chemotherapy was the strongest predictive factor for FN. The AUC improved by machine learning was 0.908 , although with a slight difference.

Chemotherapy regimen is one of the main determinants of the risk of FN as shown in the present study. In practice, some regimens are more myelotoxic than others ${ }^{4}$. Taxane- and anthracycline-based regimens were previously reported as regimens with a high risk of FN when used for the treatment of breast cancer ${ }^{4}$. CMF is less toxic than $\mathrm{AC}$ or $\mathrm{FA}(\mathrm{E}) \mathrm{C}^{3}$. Because the rates of $\mathrm{FN}$ for these and similar regimens vary considerably, it is 


\begin{tabular}{|l|l|l|l|l|l|l|}
\hline & LR & DT & XGboosting & LASSO & SVM & ANN \\
\hline AUC & 0.870 & 0.855 & 0.908 & 0.862 & 0.880 & 0.865 \\
\hline Accuracy & 0.781 & 0.759 & 0.816 & 0.805 & 0.782 & 0.782 \\
\hline Sensitivity & 0.878 & 0.707 & 0.829 & 0.805 & 0.829 & 0.854 \\
\hline Specificity & 0.696 & 0.804 & 0.804 & 0.804 & 0.739 & 0.717 \\
\hline PPV & 0.720 & 0.763 & 0.791 & 0.786 & 0.739 & 0.729 \\
\hline NPV & 0.865 & 0.755 & 0.841 & 0.822 & 0.829 & 0.846 \\
\hline
\end{tabular}

Table 3. Performance of machine learning algorithms for the prediction of febrile neutropenia. $L R$ logistic regression, $D T$ decision tree, $L A S S O$ least absolute shrinkage and selection operator, $S V M$ support vector machine, $A N N$ artificial neural network, $A U C$ area under the curve, $P P V$ positive predictive value, $N P V$ negative predictive value.

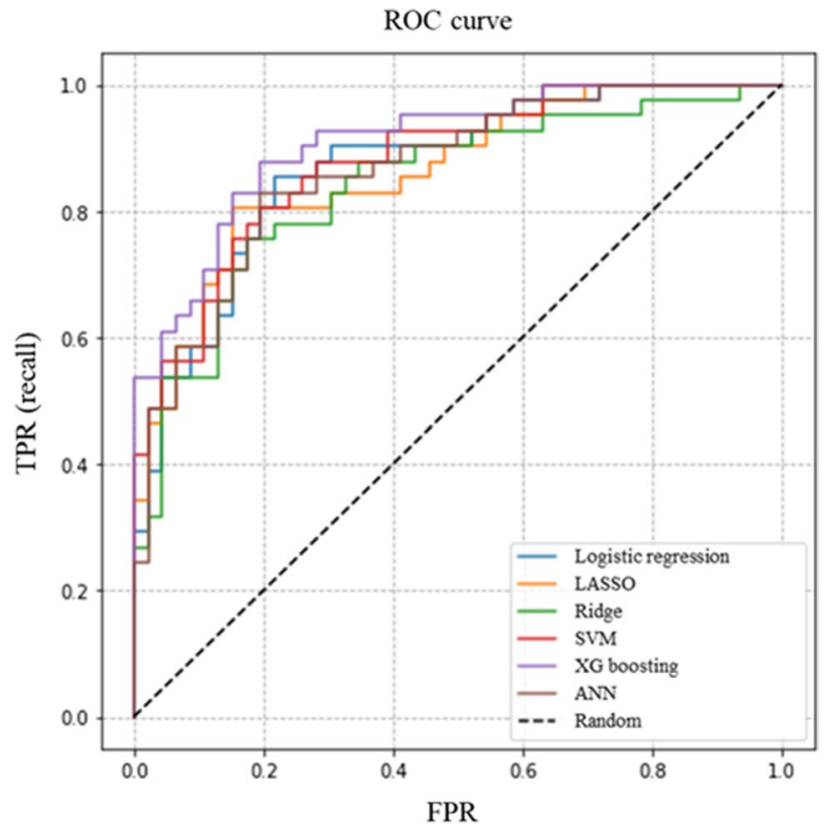

Figure 1. The AUC of each algorithm shown using colored lines. The image was drawn in Python 3.6. AUC area under the curve, $R O C$ receiver operating characteristic, TPR true positive rate, LASSO least absolute shrinkage and selection operator regression, SVM support vector machine, $A N N$ artificial neutral network.

difficult to determine the actual risk ${ }^{15}$. In addition to the regimen-specific risks, evaluating the individual risk factors in each patient can be valuable in determining appropriate treatment ${ }^{16}$.

The cycle number of the current round of chemotherapy is an important factor for FN, although only the first cycle was investigated in the present study ${ }^{11}$. Previous studies have demonstrated that the first cycle of chemotherapy is related with a greater risk for the development of FN than subsequent cycles ${ }^{2,17}$. The decreased risk of FN after subsequent cycles may be the result of clinicians' understanding of the nadir of blood counts and clinical features of patients during the first round of chemotherapy. The history of FN in a patient is a generally recognized risk factor for the development of $\mathrm{FN}^{11,18}$. Guidelines recommend the use of G-CSF as secondary prophylaxis in patients who develop FN during the equitoxic chemotherapy regimen, considering the patient's prior tolerance to chemotherapy ${ }^{11}$.

Blood counts may indicate comorbid conditions, the extent of disease, or individual response to cytotoxic chemotherapy. Even in the present study, the lymphocyte count 5 days after chemotherapy was the strongest predictive factor for FN. The slow decrease of the nadir of the lymphocyte count is apparently protective against $\mathrm{FN}^{19}$. Higher lymphocyte counts 5 days after chemotherapy may reflect higher resistance to infection, as these patients may have the potential to activate their cellular or humoral immunity rapidly ${ }^{19-21}$. However, the explicit role of lymphocytes in the development of FN remains to be elucidated.

Previous studies have reported that prophylactically administered G-CSF is significantly related with a lower risk of $\mathrm{FN}^{10,22}$. Primary prophylaxis with G-CSF can decrease the need for dose delay or reduction, antibiotics, and hospital admission ${ }^{4,18}$. Moreover, prophylactic G-CSF reduces early death, including infection-related mortality ${ }^{10,22}$. Currently, guidelines recommend prophylaxis with G-CSF when the FN risk is high (>20\%) on the basis of either chemotherapy regimen alone (high-risk regimen) or the combination of chemotherapy regimen (intermediate-risk regimen with $10-20 \% \mathrm{FN}$ risk) and personal risk factors ${ }^{4,9}$. 


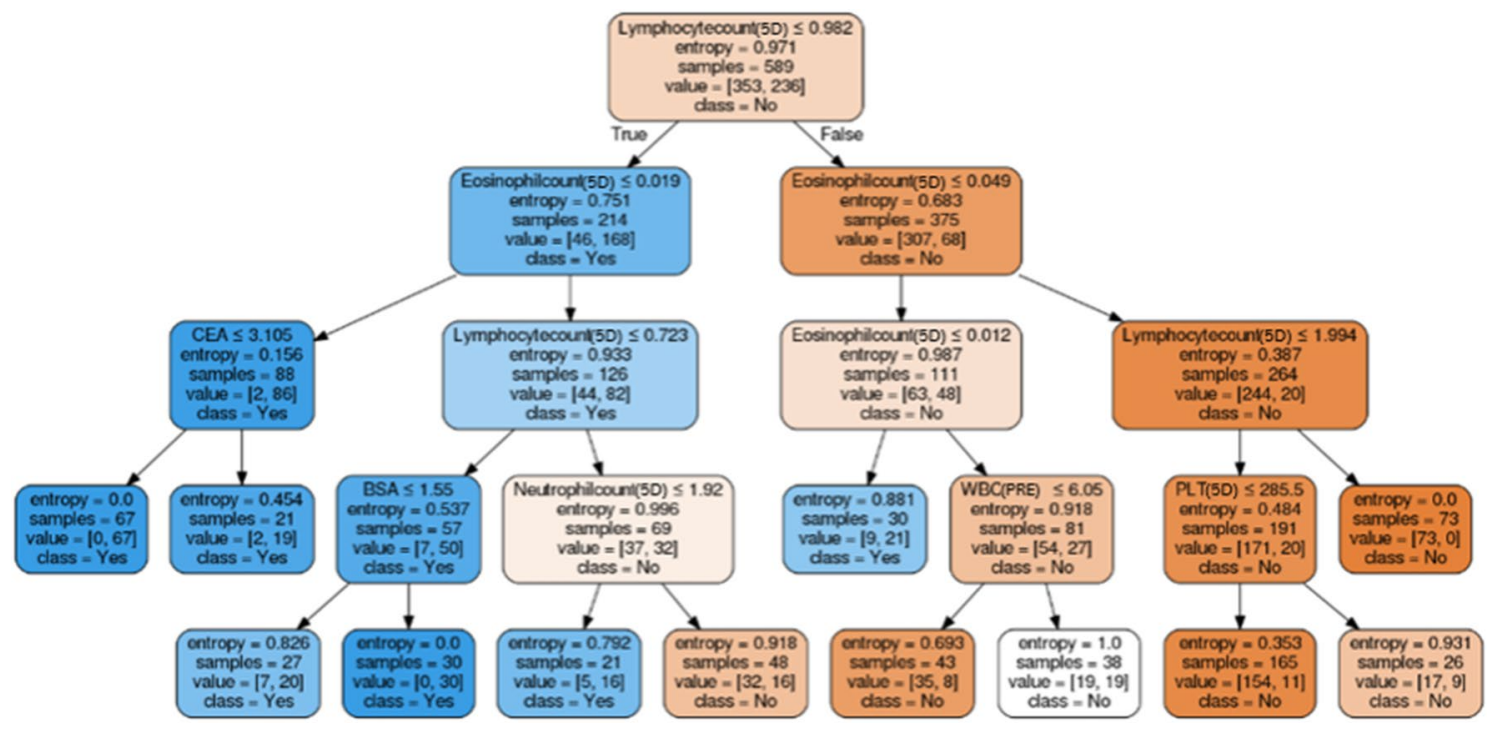

Figure 2. Detailed cut-off values displayed in a decision tree model. The image was drawn in Python 3.6. 5D 5 days after chemotherapy, $C E A$ carcinoembryonic antigen, $B S A$ body surface area, $W B C$ white blood cell, $P R E$ pretreatment, PLT platelet.

In this study, some machine learning algorithms outperformed logistic regression. This phenomenon has been observed in many prediction models using machine learning ${ }^{13,14}$. Logistic regression models are an extension of linear models using logit function as a link. Therefore, a non-linear interaction between associated factors and the outcome may not be fitted optimally. Using non-linear functions, machine learning recognizes the patterns present in the medical data and predicts the outcomes by minimizing the error ${ }^{23}$.

Our machine learning algorithm can be implemented in a clinical workflow to bridge the gap between research and practice. Considering that the period until the development of FN was $10.2 \pm 2.8$ days in our study, clinicians may use parameters including the complete blood count/differential blood count 5 days after chemotherapy to decide whether to use prophylactic G-CSF. Therefore, we envision a software tool for the prediction of FN after chemotherapy in patients with breast cancer (Supplementary Fig. S1). The software provides the predicted probability of $\mathrm{FN}$ if parameters regarding $\mathrm{FN}$ are entered using a user-friendly interface.

To the best of our knowledge, this study is the first to improve the prediction of FN after chemotherapy in patients with breast cancer by machine learning. Our predictive model defines the risk of FN after chemotherapy. The current model represents progress in predicting FN and optimizing protection against its development. This machine learning model has the potential to become a routine tool in daily clinical practice to guide the use of prophylactic G-CSF.

The present study has some limitations. First, our data showed the high rate of FN, considering that the relevant literature reported the incidence of $\mathrm{FN}$ as $10-50 \%^{8,9,24-28}$. In the current study, inpatients were purely selected because they had more lucid serial data. However, hospitalized patients usually have more severe status rather than outpatients, which can cause a selection bias. Moreover, according to the criteria of our national health insurance coverage G-CSF should be given at less than 500 of neutrophils or at less than 1,000 of neutrophils if patients have fever. Therefore, generalization should be avoided. Second, only the first cycle of each regimen was investigated. Subsequent cycles were not regarded as independent since FN may be affected by the accumulation of drugs during previous cycles. Thus, a more customized model needs to be developed for the subsequent cycles. Lastly, the decision to use G-CSF was not analyzed. Regarding the use of G-CSF, cost and national insurance coverage should be considered jointly.

In conclusion, machine learning improved the prediction of $\mathrm{FN}$ in patients undergoing chemotherapy for breast cancer. In these high-risk patients, primary prophylaxis with G-CSF could be considered. With this strategy, patient safety could be ensured during chemotherapy in patients with breast cancer.

\section{Methods}

Study design. Medical records of 1,105 hospitalized patients diagnosed with breast cancer between May 2002 and September 2018 in the Department of Breast and Endocrine surgery, Hallym University Sacred Heart Hospital were selectively reviewed for inclusion. Among them, 1,079 patients underwent surgery and were confirmed pathologically as having breast cancer. Finally, of the 1,079 patients, 933 who received chemotherapy after surgery were included in this study (Fig. 3). All patients received the first cycle of full-dose chemotherapy in the hospital, and biometric data were recorded during the treatment period. This study was approved by the Institutional Review Board of Hallym University Sacred Heart Hospital (No. 2018-04-018) and adhered to the tenets of the Helsinki Declaration. The requirement for written informed consent was waived by the Institutional Review Board. 
1,105 hospitalized patients with WHO code (C50)

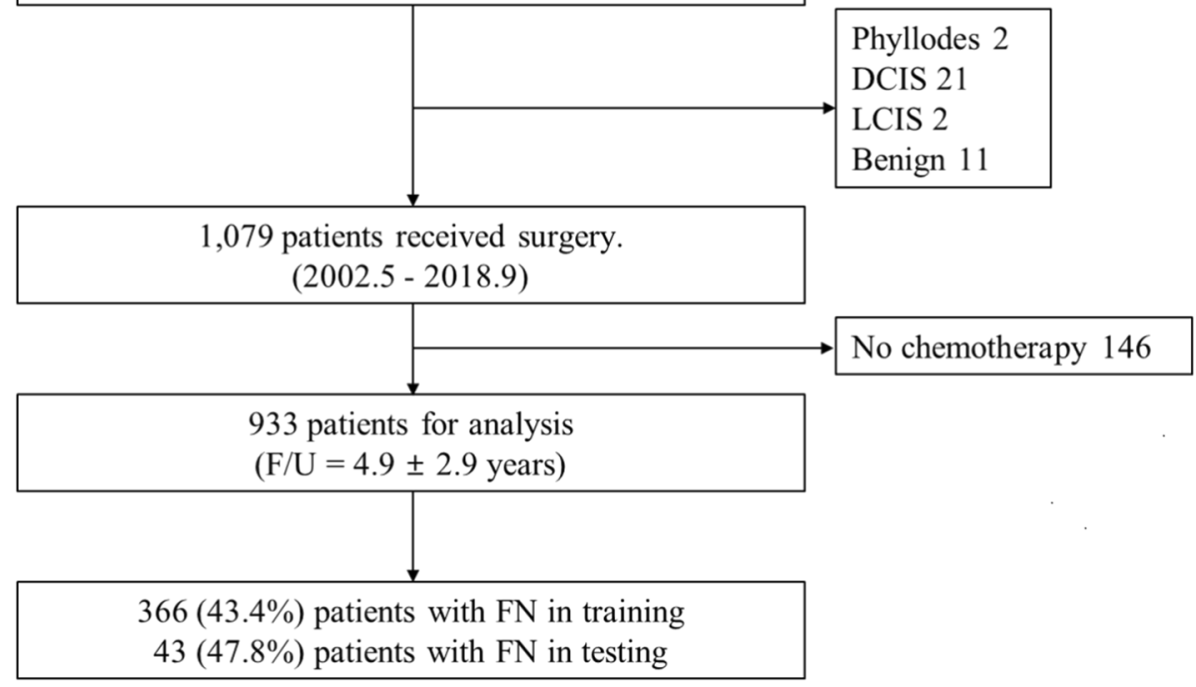

Figure 3. Flow diagram depicting the study design. The image was drawn in Microsoft PowerPoint 2016. WHO World Health Organization, DCIS ductal carcinoma in situ, LCIS lobular carcinoma in situ, F/U follow-up, FN febrile neutropenia.

Datasets. The entire cohort was divided into a training and testing dataset which were mutually exclusive. The training dataset was built with 843 patients treated between May 2002 and January 2018. The testing dataset consisted of 90 patients treated between February 2018 and September 2018 and was used to validate the performance of machine learning models. In both datasets, patients who had any missing data for clinical, pathological, or therapeutic variables of interest were excluded from the analyses.

Assessments. Demographic, clinical, pathological, and therapeutic information were obtained from the medical records of study participants. Tumors were staged according to the 8th edition of the American Joint Committee on Cancer staging system. FN was defined as the incidence of fever of $38.3^{\circ} \mathrm{C}$ or $38.0^{\circ} \mathrm{C}$ for over $1 \mathrm{~h}$ orally, and neutrophil count $<500$ or $500-999 / \mathrm{mm}^{3}$ with predicted drop to $<500 / \mathrm{mm}^{3}$ over next $48 \mathrm{~h}^{9}$. During the first cycle of chemotherapy, each patient was monitored carefully for the development of FN.

Analysis. To extract the factors associated with FN, classical and recent machine learning algorithms were applied. Least absolute shrinkage and selection operator regression, ridge regression, support vector machine, decision tree, XGboosting, and artificial neural network were used for machine learning algorithms. Conventional stepwise logistic regression was used as a reference method. Factors associated with FN were selected from the dataset using the recursive feature elimination $\operatorname{method}^{29}$. The $p$ value used to select and remove the factor in the forward stepwise process was 0.05 . Factor selection and model construction were done on the platform with scikit-learn 0.20 in Python 3.6 (Python Software Foundation, Wilmington, DE). Prediction models were constructed for each machine learning algorithm with the training dataset using the optimal feature subset for each machine-learning algorithm. Five-fold cross-validation was used for evaluation. The performance of the prediction models was evaluated in the testing dataset. The area under the curve (AUC) was used as the main measurement.

\section{Data availability}

All the data supporting the findings of this study are available from the corresponding author upon reasonable request.

Received: 18 December 2019; Accepted: 24 August 2020

Published online: 09 September 2020

\section{References}

1. de Naurois, J. et al. Management of febrile neutropenia: ESMO Clinical Practice Guidelines. Ann. Oncol. 21, v252-v256 (2010).

2. Hosmer, W., Malin, J. \& Wong, M. Development and validation of a prediction model for the risk of developing febrile neutropenia in the first cycle of chemotherapy among elderly patients with breast, lung, colorectal, and prostate cancer. Support. Care Cancer 19, 333-341 (2011).

3. Crawford, J., Dale, D. C. \& Lyman, G. H. Chemotherapy-induced neutropenia: risks, consequences, and new directions for its management. Cancer 100, 228-237 (2004). 
4. Aapro, M. S. et al. 2010 update of EORTC guidelines for the use of granulocyte-colony stimulating factor to reduce the incidence of chemotherapy-induced febrile neutropenia in adult patients with lymphoproliferative disorders and solid tumours. Eur. J. Cancer 47, 8-32 (2011).

5. Kuderer, N. M., Dale, D. C., Crawford, J., Cosler, L. E. \& Lyman, G. H. Mortality, morbidity, and cost associated with febrile neutropenia in adult cancer patients. Cancer 106, 2258-2266 (2006).

6. Lyman, G. H. et al. Predicting individual risk of neutropenic complications in patients receiving cancer chemotherapy. Cancer 117, 1917-1927 (2011).

7. Chang, J. Chemotherapy dose reduction and delay in clinical practice. Evaluating the risk to patient outcome in adjuvant chemotherapy for breast cancer. Eur. J. Cancer 36, S11-S14 (2000).

8. Bennett, C. L., Djulbegovic, B., Norris, L. B. \& Armitage, J. O. Colony-stimulating factors for febrile neutropenia during cancer therapy. N. Engl. J. Med. 368, 1131-1139 (2013).

9. Crawford, J. et al. Myeloid growth factors, Version 2.2017, NCCN Clinical Practice Guidelines in Oncology. J. Natl. Compr. Canc. Netw. 15, 1520-1541 (2017).

10. Herbst, C. et al. Prophylactic antibiotics or G-CSF for the prevention of infections and improvement of survival in cancer patients undergoing chemotherapy. Cochrane Database Syst. Rev. 1, CD007107 (2009).

11. Bozcuk, H. et al. A prospectively validated nomogram for predicting the risk of chemotherapy-induced febrile neutropenia: a multicenter study. Support. Care Cancer 23, 1759-1767 (2015).

12. Lyman, G. H., Lyman, C. H. \& Agboola, O. Risk models for predicting chemotherapy-induced neutropenia. Oncologist 10, 427-437 (2005).

13. Mirza, B. et al. Machine learning and integrative analysis of biomedical big data. Genes 10, 87 (2019).

14. Low, S. K., Zembutsu, H. \& Nakamura, Y. Breast cancer: The translation of big genomic data to cancer precision medicine. Cancer Sci. 109, 497-506 (2018).

15. Dale, D. C., McCarter, G. C., Crawford, J. \& Lyman, G. H. Myelotoxicity and dose intensity of chemotherapy: reporting practices from randomized clinical trials. J. Natl. Compr. Canc. Netw. 1, 440-454 (2003).

16. Dranitsaris, G. et al. Identifying patients at high risk for neutropenic complications during chemotherapy for metastatic breast cancer with doxorubicin or pegylated liposomal doxorubicin: the development of a prediction model. Am. J. Clin. Oncol. 31, 369-374 (2008).

17. Crawford, J. et al. Risk and timing of neutropenic events in adult cancer patients receiving chemotherapy: the results of a prospective nationwide study of oncology practice. J. Natl. Compr. Canc. Netw. 6, 109-118 (2008).

18. Kelly, S. \& Wheatley, D. Prevention of febrile neutropenia: use of granulocyte colony-stimulating factors. Br. J. Cancer 101, S6-S10 (2009).

19. Choi, C. W. et al. Early lymphopenia as a risk factor for chemotherapy-induced febrile neutropenia. Am. J. Hematol. 73, 263-266 (2003).

20. Blay, J. Y. et al. Early lymphopenia after cytotoxic chemotherapy as a risk factor for febrile neutropenia. J. Clin. Oncol. 14, 636-643 (1996).

21. Ray-Coquard, I. et al. Baseline and early lymphopenia predict for the risk of febrile neutropenia after chemotherapy. Br. J. Cancer 88, 181-186 (2003).

22. Kuderer, N. M., Dale, D. C., Crawford, J. \& Lyman, G. H. Impact of primary prophylaxis with granulocyte colony-stimulating factor on febrile neutropenia and mortality in adult cancer patients receiving chemotherapy: a systematic review. J. Clin. Oncol. 25, 3158-3167 (2007).

23. Dreiseitl, S. \& Ohno-Machado, L. Logistic regression and artificial neural network classification models: a methodology review. J. Biomed. Inform. 35, 352-359 (2002).

24. White, L. \& Ybarra, M. Neutropenic Fever. Hematol. Oncol. Clin. North. Am. 31, 981-993 (2017).

25. Engert, A. et al. Incidence of febrile neutropenia and myelotoxicity of chemotherapy: a meta-analysis of biosimilar G-CSF studies in breast cancer, lung cancer, and non-Hodgkin's lymphoma. Onkologie 32, 599-604 (2009).

26. Aapro, M. S. et al. EORTC guidelines for the use of granulocyte-colony stimulating factor to reduce the incidence of chemotherapyinduced febrile neutropenia in adult patients with lymphomas and solid tumours. Eur. J. Cancer 42, 2433-2453 (2006).

27. Lyman, G. H., Kuderer, N. M. \& Djulbegovic, B. Prophylactic granulocyte colony-stimulating factor in patients receiving doseintensive cancer chemotherapy: a meta-analysis. Am. J. Med. 112, 406-411 (2002).

28. Caggiano, V., Weiss, R. V., Rickert, T. S. \& Linde-Zwirble, W. T. Incidence, cost, and mortality of neutropenia hospitalization associated with chemotherapy. Cancer 103, 1916-1924 (2005).

29. Romero, E. \& Sopena, J. M. Performing feature selection with multilayer perceptrons. IEEE Trans. Neural Netw. 19, 431-441 (2008).

\section{Acknowledgements}

This research was supported by the Bio \& Medical Technology Development Program of the National Research Foundation (NRF), and the Korean government fund (MSIT) (No. NRF-2017M3A9E8033207, 2019R1G1A1004679).

\section{Author contributions}

B.C. contributed to data analysis, manuscript preparation, and Figs. (2 and 3). K.K. contributed to data analysis and manuscript review. S.B. contributed to data collection and manuscript review. Y.S. contributed to study design, data analysis, manuscript preparation, and Figs. (1 and S1). All authors read and approved the final manuscript.

\section{Competing interests}

The authors declare no competing interest.

\section{Additional information}

Supplementary information is available for this paper at https://doi.org/10.1038/s41598-020-71927-6.

Correspondence and requests for materials should be addressed to Y.J.S.

Reprints and permissions information is available at www.nature.com/reprints.

Publisher's note Springer Nature remains neutral with regard to jurisdictional claims in published maps and institutional affiliations. 
(c) (i) Open Access This article is licensed under a Creative Commons Attribution 4.0 International cc) License, which permits use, sharing, adaptation, distribution and reproduction in any medium or format, as long as you give appropriate credit to the original author(s) and the source, provide a link to the Creative Commons licence, and indicate if changes were made. The images or other third party material in this article are included in the article's Creative Commons licence, unless indicated otherwise in a credit line to the material. If material is not included in the article's Creative Commons licence and your intended use is not permitted by statutory regulation or exceeds the permitted use, you will need to obtain permission directly from the copyright holder. To view a copy of this licence, visit http://creativecommons.org/licenses/by/4.0/.

(C) The Author(s) 2020 